\begin{tabular}{|c|c|}
\hline Title & Stochastic phase transition operator \\
\hline Author(s) & Y amanobe, Takanobu \\
\hline Citation & $\begin{array}{l}\text { Physical Review E, 84(1), } 011924 \\
\text { https://doi.org/10.1103/PhysRevE.84.011924 }\end{array}$ \\
\hline Issue Date & 2011-07 \\
\hline Doc URL & http:/hdl. handle.net/2115/46957 \\
\hline Rights & @2011 A merican Physical Society \\
\hline Type & article \\
\hline File Information & PRE84_1_011924.pdf \\
\hline
\end{tabular}

Instructions for use 


\title{
Stochastic phase transition operator
}

\author{
Takanobu Yamanobe* \\ Hokkaido University School of Medicine, North 15, West 7, Kita-ku, Sapporo 060-8638, Japan and Precursory Research for Embryonic \\ Science and Technology, Japan Science and Technology Agency, 4-1-8 Honcho Kawaguchi, Saitama 332-0012, Japan
}

(Received 7 January 2011; revised manuscript received 18 April 2011; published 26 July 2011)

\begin{abstract}
In this study a Markov operator is introduced that represents the density evolution of an impulse-driven stochastic biological oscillator. The operator's stochastic kernel is constructed using the asymptotic expansion of stochastic processes instead of solving the Fokker-Planck equation. The Markov operator is shown to successfully approximate the density evolution of the biological oscillator considered. The response of the oscillator to both periodic and time-varying impulses can be analyzed using the operator's transient and stationary properties. Furthermore, an unreported stochastic dynamic bifurcation for the biological oscillator is obtained by using the eigenvalues of the product of the Markov operators.
\end{abstract}

DOI: 10.1103/PhysRevE.84.011924

PACS number(s): 87.10.Mn, 87.19.1c, 05.10.Gg, 02.50.Fz

\section{INTRODUCTION}

The convergence of a system to asymptotic dynamics such as the equilibrium point, limit cycle, and chaos often requires more time than the time scale of realistic phenomena of the system. Examples of systems showing slow convergence are nervous and ecological systems [1-4]. In such a situation of slow convergence, the system behavior can be better understood using its transient dynamics than its asymptotic dynamics. In many cases, stochasticity greatly improves the lifetime of a transient [4-6]. This highlights the importance of transient dynamics in noisy systems such as nervous systems.

A neuron or neural network is in the transient regime if its input changes much faster than the convergence speed to its asymptotic dynamics. Transient dynamics are also related to the following problem of the information carriers in nervous systems. Spike sequences are key information carriers in nervous systems; however, the exact spike statistic that is the information carrier remains unclear [7]. Since spikes contain encoded information in the transient regime, transient dynamics of neurons and neural networks are important for understanding the coding scheme. Several studies have actually found that neurons and neural networks function in the transient regime; examples of findings include the transition of several response regimes in pacemaker neurons in response to time-varying synaptic inputs $[1,8]$ and the sensitive dependence of neuronal activity on the stimulus in an olfactory system [2]. Since transient dynamics can occur far from the invariant structure of a system, it is necessary to understand the dynamics of the entire phase space.

One possible way to analyze the dynamics of the entire phase space is to use Markov operators, which are linear integral operators that can be applied to describe the evolution of densities corresponding to given stochastic differential equations $[9,10]$. Markov operators have a wide range of applications; for example, they can be applied to analyses of coupled map lattices [11,12], nonlinear oscillators driven by stochastic inputs $[5,6,13,14]$, neuronal dynamics [15-21], and neural networks [22].

\footnotetext{
*yamanobe@med.hokudai.ac.jp
}

Markov operators can be constructed using stochastic kernels, which are transition densities corresponding to the given stochastic differential equations. In this case, the transition densities are governed by the corresponding Fokker-Planck equation. This equation can be solved analytically for some cases; however, for most cases, solving it is generally very difficult [23]. This difficulty can be avoided by either assuming a known transition density [18] or using the first passage time approach $[5,6,13,17,20]$. However, these approaches have a limited range of application and this restricts the applicability of Markov operators.

In this study a Markov operator of an impulse-driven stochastic biological oscillator is introduced whose deterministic version is called a radial isochron clock or Poincaré oscillator, one of the canonical models of neuronal oscillators [1,18,19,24-26]. Furthermore, the small-disturbance asymptotic theory [27] is applied, which is a theory of asymptotic expansion of stochastic processes, to calculate the transition densities. Using this theory, the construction of a Markov operator that governs the density evolution of the impulse-driven stochastic biological oscillator is shown. It is also shown that the product of the Markov operators can approximate the density evolution of oscillators driven by time-varying impulses far from the limit cycle of the oscillator. The eigenvalues and eigenfunctions of the Markov operator are used to analyze the dynamics of the stochastic oscillator driven by periodic impulses. These analyzed dynamics are then used to analyze the oscillator dynamics in response to time-varying impulses. An unreported stochastic bifurcation is obtained in the case of time-varying impulses on the basis of eigenvalues of the product of the Markov operators and components governing the transient dynamics of neurons are discussed.

This paper is organized as follows. Section II presents the stochastic biological oscillator used in this study. Section III introduces the Markov operator that represents the evolution of the densities of this oscillator. Section IV summarizes the properties of the Markov operator and presents a derivation of the properties of the stochastic biological oscillator in response to time-varying impulses. Section V describes a numerical analysis of the oscillator in response to periodic impulses and timevarying impulses using the Markov operator. Finally, Sec. VI discusses the results in relation to the neural coding problem and the generalization of the operator constructed in this study. 


\section{STOCHASTIC BIOLOGICAL OSCILLATOR}

An impulse-driven stochastic biological oscillator is expressed as

$$
\begin{aligned}
d X_{t}^{(\epsilon)}= & {\left[k\left\{1-\left(X_{t}^{(\epsilon)^{2}}+Y_{t}^{(\epsilon)^{2}}\right)^{1 / 2}\right\} X_{t}^{(\epsilon)}-2 \pi Y_{t}^{(\epsilon)}\right.} \\
& \left.+\sum_{t_{n}<t} A_{n} \delta\left(t-t_{n}\right)\right] d t+\epsilon d W_{t}, \\
d Y_{t}^{(\epsilon)}= & {\left[k\left\{1-\left(X_{t}^{(\epsilon)^{2}}+Y_{t}^{(\epsilon)^{2}}\right)^{1 / 2}\right\} Y_{t}^{(\epsilon)}+2 \pi X_{t}^{(\epsilon)}\right] d t, }
\end{aligned}
$$

where $X_{t}^{(\epsilon)}$ and $Y_{t}^{(\epsilon)}$ denote the membrane potential and refractoriness, respectively, and their superscript and subscript represent the dependence of the random variables on the parameter $\epsilon \in(0,1]$ and time $t$, respectively. $k$ is a positive parameter and $W_{t}$ is a one-dimensional standard Wiener process. The $n$th impulse is added at time $t_{n}(n=1,2, \ldots)$ with amplitude $A_{n}$; the $n$th interimpulse interval is defined as $I_{n}=t_{n+1}-t_{n}$. Without the impulse term Eq. (1) is called a stochastic Poincaré oscillator in this paper since the deterministic version is called a Poincaré oscillator [24].

The solution of Eq. (1) between the $n$th and $(n+1)$ th impulses is considered. In this case Eq. (1) needs to be considered without the impulses term. First, the equation is translated into the polar coordinate system, i.e., $R_{t}^{(\epsilon)}=$ $\left(X_{t}^{(\epsilon)^{2}}+Y_{t}^{(\epsilon)^{2}}\right)^{1 / 2}$ and $\Phi_{t}^{(\epsilon)}=(2 \pi)^{-1} \arctan \left(Y_{t}^{(\epsilon)} / X_{t}^{(\epsilon)}\right)$, where the latter is a normalized angular coordinate varying in $[0,1)$, to solve Eq. (1) analytically. In this case Eq. (1) is written in the Ito formulation as follows (see Ito's formula for this translation in Ref. [28]):

$$
\begin{aligned}
d R_{t}^{(\epsilon)}= & k R_{t}^{(\epsilon)}\left(1-R_{t}^{(\epsilon)}\right) d t+\frac{\epsilon^{2}}{2} \frac{\sin ^{2}\left(2 \pi \Phi_{t}^{(\epsilon)}\right)}{R_{t}^{(\epsilon)}} d t \\
& +\epsilon \cos \left(2 \pi \Phi_{t}^{(\epsilon)}\right) d W_{t} \\
d \Phi_{t}^{(\epsilon)}= & d t+\frac{\epsilon^{2}}{4 \pi} \frac{\sin \left(4 \pi \Phi_{t}^{(\epsilon)}\right)}{R_{t}^{(\epsilon)^{2}}} d t-\frac{\epsilon}{2 \pi} \frac{\sin \left(2 \pi \Phi_{t}^{(\epsilon)}\right)}{R_{t}^{(\epsilon)}} d W_{t}
\end{aligned}
$$

The solution of Eq. (2) with $\epsilon=0$ is considered. First the initial conditions (Fig. 1) are considered. According to Ref. [24], the $n$th impulse with amplitude $A_{n}$ shifts a state point $(r, \phi)$ to $\left(r^{\prime}, \phi^{\prime}\right)$, where

$$
\begin{gathered}
r^{\prime}=\left\{r^{2}+A_{n}^{2}+2 A_{n} r \cos (2 \pi \phi)\right\}^{1 / 2}, \\
\phi^{\prime}=(2 \pi)^{-1} \arccos \left\{\left(r \cos (2 \pi \phi)+A_{n}\right) / r^{\prime}\right\} .
\end{gathered}
$$

The preceding arc-cosine function must be evaluated as $0<\phi^{\prime}<0.5$ for $0<\phi<0.5$ and $0.5<\phi^{\prime}<1$ for $0.5<\phi<1$. This relation corresponds to the two-dimensional version of the phase transition curve of this oscillator, which represents the phase shift due to a single isolated impulse. Similarly, according to Ref. [24], a trajectory starting from $\left(r^{\prime}, \phi^{\prime}\right)$, which is just after the $n$th impulse, after a time interval $I_{n}$ is expressed as follows:

$$
\begin{aligned}
R_{I_{n}}^{(0)}= & r^{\prime} /\left\{\left(1-r^{\prime}\right) e^{-k I_{n}}+r^{\prime}\right\}, \\
& \Theta_{I_{n}}^{(0)}=\phi^{\prime}+I_{n},
\end{aligned}
$$

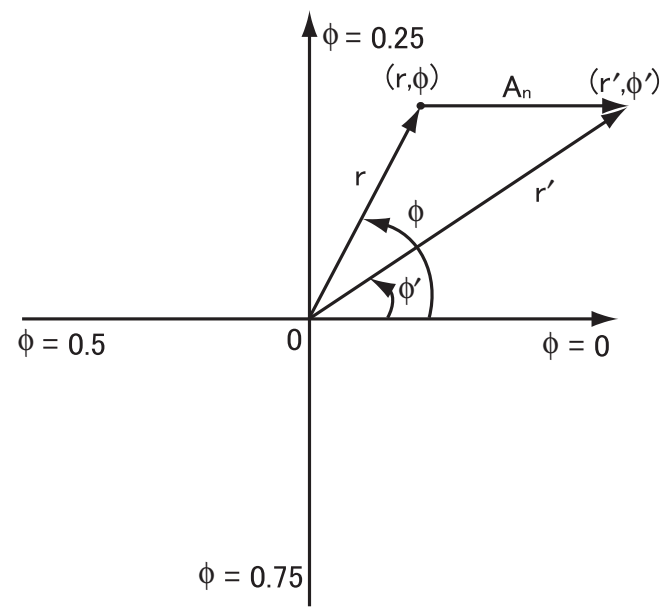

FIG. 1. Effect of an impulse on a state point of a stochastic Poincaré oscillator. The state point $(r, \phi)$ is displaced by the amount $A_{n}$ in the $X$ direction.

where $\Phi_{I_{n}}^{(0)}=\Theta_{I_{n}}^{(0)} \quad(\bmod 1)$. In general, $\Phi_{t}^{(\epsilon)}=\Theta_{t}^{(\epsilon)}$ (mod 1); that is, $\Theta_{t}^{(\epsilon)}$ takes a value in $\boldsymbol{R}$ and explicitly indicates the rotation around the origin.

\section{STOCHASTIC PHASE TRANSITION OPERATOR}

Based on the preceding background, a Markov operator is constructed that relates the density just before the $n$th impulse to that just before the $(n+1)$ th impulse; namely, it relates the response of the stochastic Poincare oscillator to the $n$th impulse. The integral form of Eq. (2) with the random variable $\Theta_{t}^{(\epsilon)}$ with initial condition $\left(r^{\prime}, \phi^{\prime}\right)$ is

$$
\begin{aligned}
R_{I_{n}}^{(\epsilon)}= & r^{\prime}+k \int_{0}^{I_{n}} R_{s}^{(\epsilon)}\left(1-R_{s}^{(\epsilon)}\right) d s \\
& +\frac{\epsilon^{2}}{2} \int_{0}^{I_{n}} \frac{\sin ^{2}\left(2 \pi \Theta_{s}^{(\epsilon)}\right)}{R_{s}^{(\epsilon)}} d s+\epsilon \int_{0}^{I_{n}} \cos \left(2 \pi \Theta_{s}^{(\epsilon)}\right) d W_{s}, \\
\Theta_{I_{n}}^{(\epsilon)}= & \phi^{\prime}+I_{n}+\frac{\epsilon^{2}}{4 \pi} \int_{0}^{I_{n}} \frac{\sin \left(4 \pi \Theta_{s}^{(\epsilon)}\right)}{R_{s}^{(\epsilon)^{2}}} d s \\
& -\frac{\epsilon}{2 \pi} \int_{0}^{I_{n}} \frac{\sin \left(2 \pi \Theta_{s}^{(\epsilon)}\right)}{R_{s}^{(\epsilon)}} d W_{s} .
\end{aligned}
$$

As mentioned earlier, Markov operators can be constructed using stochastic kernels, i.e., transition densities corresponding to the given stochastic differential equations. However, the corresponding Fokker-Planck equation governing these densities is difficult to solve analytically in most cases [23]. Therefore, in what follows, an asymptotic expansion of stochastic processes, called the small-disturbance asymptotic theory [27,29], is applied to the calculation of the transition density starting from $(r, \phi)$. As explained above, the state point $(r, \phi)$ is shifted to the state point $\left(r^{\prime}, \phi^{\prime}\right)$ by the $n$th impulse. After this discontinuous shift, the dynamics of the oscillator is determined by Eq. (3). Applying the theory of the asymptotic 

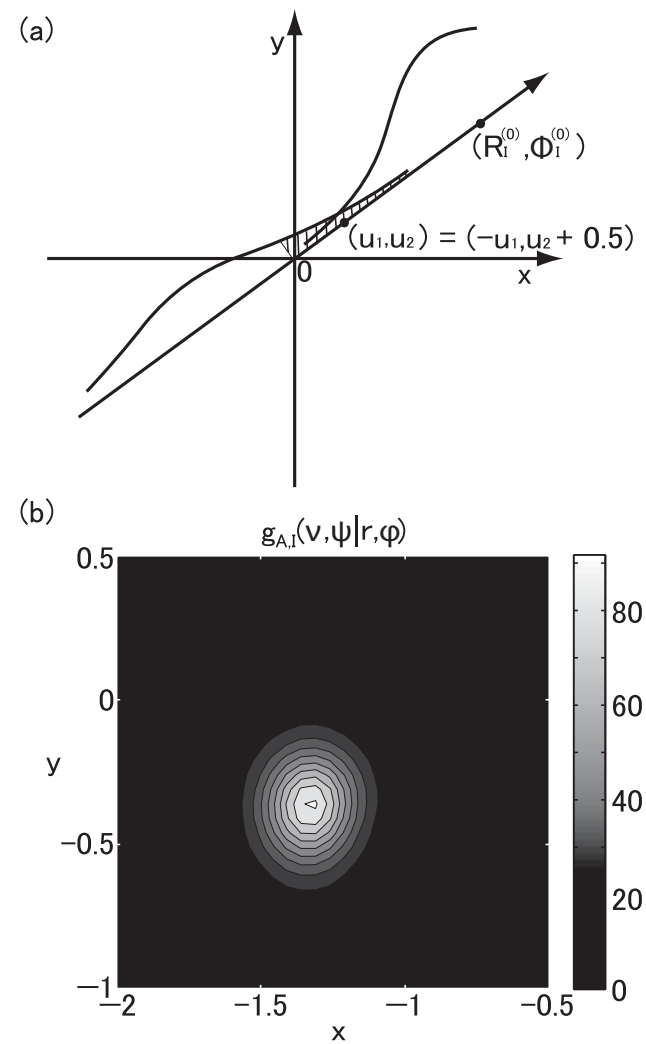

FIG. 2. (a) Construction of a stochastic kernel (see the text for more details). (b) Example of a stochastic kernel. The parameters used are $A=0.9, I=0.6, r=1.2, \phi=0.9, k=1.0$, and $\epsilon=$ 0.3. In both (a) and (b) the abscissa and ordinate are $x$ and $y$, respectively.

expansion to Eq. (3), one can avoid the discontinuity produced by the $n$th impulse and finally calculate the transition density starting from $(r, \phi)$. to $\epsilon$ as

One sets $\boldsymbol{U}_{I_{n}}^{(\epsilon)} \equiv\left(R_{I_{n}}^{(\epsilon)}, \Theta_{I_{n}}^{(\epsilon)}\right)$ and expands $\boldsymbol{U}_{I_{n}}^{(\epsilon)}$ with respect

$$
\boldsymbol{U}_{I_{n}}^{(\epsilon)}=\boldsymbol{U}_{I_{n}}^{(0)}+\epsilon \boldsymbol{A}_{1 I_{n}}+o(\epsilon)
$$

where $\boldsymbol{U}_{I_{n}}^{(0)}$ is a solution of Eq. (3) with $\epsilon=0$ and $\boldsymbol{A}_{1 I_{n}}=$ $\left(A_{1 R I_{n}}, A_{1 \Theta I_{n}}\right)=\partial \boldsymbol{U}_{I_{n}}^{(\epsilon)} /\left.\partial \epsilon\right|_{\epsilon=0}$. Here the subscripts $R$ and $\Theta$ denote the components of $\boldsymbol{A}_{1 I_{n}}$ explicitly and the subscript $I_{n}$ indicates that the corresponding variable is a function of the time interval. These components are derived as follows:

$$
\begin{aligned}
& A_{1 R t}=k \int_{0}^{t} A_{1 R s}\left(1-2 R_{s}^{(0)}\right) d s+\int_{0}^{t} \cos \left(2 \pi \Theta_{s}^{(0)}\right) d W_{s}, \\
& A_{1 \Theta t}=-\frac{1}{2 \pi} \int_{0}^{t} \frac{\sin \left(2 \pi \Theta_{s}^{(0)}\right)}{R_{s}^{(0)}} d W_{s} .
\end{aligned}
$$

These equations can be solved to obtain the components after the time interval $I_{n}$ as

$$
\begin{aligned}
A_{1 R I_{n}}= & \int_{0}^{I_{n}} \exp \left\{k\left(I_{n}-s\right)\right. \\
& \left.-2 k \int_{s}^{I_{n}} R_{u}^{(0)} d u\right\} \cos \left(2 \pi \Theta_{s}^{(0)}\right) d W_{s} \\
A_{1 \Theta I_{n}}= & -\frac{1}{2 \pi} \int_{0}^{I_{n}} \frac{\sin \left(2 \pi \Theta_{s}^{(0)}\right)}{R_{s}^{(0)}} d W_{s} .
\end{aligned}
$$

A higher-order expansion of $\boldsymbol{U}_{I_{n}}^{(\epsilon)}$ is possible and may give a better approximation.

To consider the stochastic dynamics around the solution of Eq. (3) with $\epsilon=0$, a random variable $S_{I_{n}}^{(\epsilon)}=\left(\boldsymbol{U}_{I_{n}}^{(\epsilon)}-\boldsymbol{U}_{I_{n}}^{(0)}\right) / \epsilon$ is introduced. Expansion of $\boldsymbol{S}_{I_{n}}^{(\epsilon)}$ with respect to $\epsilon$ gives

$$
S_{I_{n}}^{(\epsilon)}=A_{1 I_{n}}+o(1) .
$$

The asymptotic expansion of the characteristic function of $S_{I_{n}}^{(\epsilon)}$ with respect to $\epsilon$ is

$$
\begin{aligned}
\Psi(\boldsymbol{\xi}) & =\boldsymbol{E}\left[\exp \left\{i \boldsymbol{\xi} \cdot\left(\boldsymbol{A}_{1 I_{n}}+o(1)\right)\right\}\right] \\
& =\boldsymbol{E}\left[\exp \left(i \boldsymbol{\xi} \cdot \boldsymbol{A}_{1 I_{n}}\right)\right]+o(1) \\
& =\exp \left\{-\left(\boldsymbol{\xi} \cdot \boldsymbol{\Sigma}_{I_{n}} \boldsymbol{\xi}\right) / 2\right\}+o(1),
\end{aligned}
$$

where $\boldsymbol{\xi} \in \boldsymbol{R}^{2}$. The matrix $\boldsymbol{\Sigma}_{I_{n}}$ is constructed using $A_{1 R I_{n}}$ and $A_{1 \Theta I_{n}}$ as

$$
\begin{aligned}
\boldsymbol{\Sigma}_{I_{n}}= & \left(\begin{array}{lc}
\boldsymbol{E}\left[A_{1 R I_{n}}^{2}\right] & \boldsymbol{E}\left[A_{1 R I_{n}} A_{1 \Theta I_{n}}\right] \\
\boldsymbol{E}\left[A_{1 R I_{n}} A_{1 \Theta I_{n}}\right] & \boldsymbol{E}\left[A_{1 \Theta I_{n}}^{2}\right]
\end{array}\right), \\
\boldsymbol{E}\left[A_{1 R I_{n}}^{2}\right]= & \int_{0}^{I_{n}} \exp \left\{2 k\left(I_{n}-s\right)-4 k \int_{s}^{I_{n}} R_{u}^{(0)} d u\right\} \\
& \times \cos ^{2}\left(2 \pi \Theta_{s}^{(0)}\right) d s, \\
\boldsymbol{E}\left[A_{1 \Theta I_{n}}^{2}\right]= & \frac{1}{(2 \pi)^{2}} \int_{0}^{I_{n}} \frac{\sin ^{2}\left(2 \pi \Theta_{s}^{(0)}\right)}{R_{s}^{(0)^{2}}} d s,
\end{aligned}
$$

$$
\boldsymbol{E}\left[A_{1 R I_{n}} A_{1 \Theta I_{n}}\right]=-\frac{1}{2 \pi} \int_{0}^{I_{n}} \frac{\exp \left\{k\left(I_{n}-s\right)-2 k \int_{s}^{I_{n}} R_{u}^{(0)} d u\right\} \cos \left(2 \pi \Theta_{s}^{(0)}\right) \sin \left(2 \pi \Theta_{s}^{(0)}\right)}{R_{s}^{(0)}} d s
$$

It is noteworthy that these components can be solved analytically. In Eq. (5) the second equality is derived from the expansion of the exponential function with respect to $\epsilon$. The third equality is derived from the Gaussianity of $A_{1 R I_{n}}$ and $A_{1 \Theta I_{n}}$. Using the inverse of this characteristic function, one obtains

$$
f_{\boldsymbol{S}_{I_{n}}^{(\epsilon)}}(\boldsymbol{u})=n\left[\boldsymbol{u} ; \mathbf{0}, \boldsymbol{\Sigma}_{I_{n}}\right]+o(1),
$$

where $\boldsymbol{u}=\left(u_{1}, u_{2}\right) \in \boldsymbol{R}^{2}, \mathbf{0}=(0,0) \in \boldsymbol{R}^{2}$, and $n\left[\boldsymbol{u} ; \mathbf{0}, \boldsymbol{\Sigma}_{I_{n}}\right]$ is the density of a two-dimensional Gaussian distribution with zero mean and the variance-covariance matrix $\boldsymbol{\Sigma}_{I_{n}}$. Equation (6) is derived by expansion around the solution $\boldsymbol{U}_{I_{n}}^{(0)}$. The obtained density expressed in Eq. (6) is the two-dimensional Gaussian distribution in the directions 

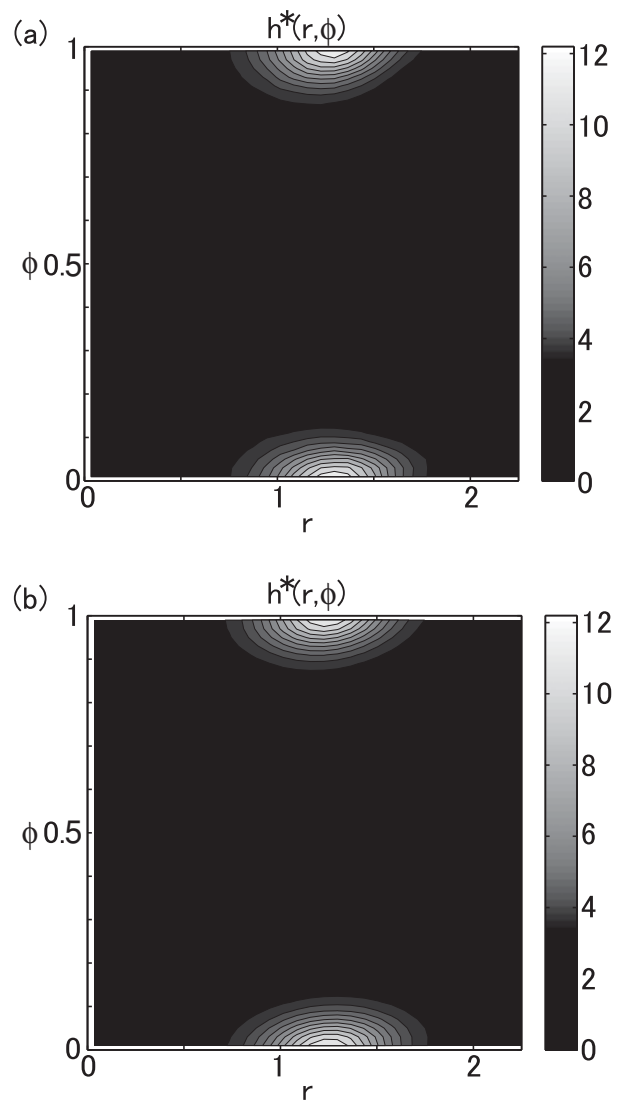

FIG. 3. Comparison of invariant densities obtained by the (a) Euler method and (b) SPTO. Both densities are plotted in the $r-\phi$ system (i.e., polar coordinate system) for comparison. The parameters used are $\epsilon=0.6, A=0.9, I=1.0$, and $k=1.0$. For case (a), the time step is 0.0001 , the number of impulses is 50 , and the number of different sample paths used is 500000 .

of $R$ and $\Theta$; that is, $u_{1}$ and $u_{2}$ are coordinates in the directions of $R$ and $\Theta$, respectively. The first term of Eq. (6) is used to approximate the density of $\boldsymbol{U}_{I_{n}}^{(\epsilon)}$ as follows:

$$
f_{\boldsymbol{U}_{I_{n}}^{(\epsilon)}}(\boldsymbol{u}) \sim n\left[\boldsymbol{u} ; \boldsymbol{U}_{I_{n}}^{(0)}, \epsilon^{2} \boldsymbol{\Sigma}_{I_{n}}\right]
$$

Equation (7) is used to construct the stochastic kernel; however, $r^{\prime}=0$ is not used in the construction. Since $u_{1}$ and $u_{2}$ are coordinates in the directions of $R$ and $\Theta$, respectively, point $\left(u_{1}, u_{2}\right)$ is equal to point $\left(-u_{1}, u_{2}+0.5\right)$ [Fig. 2(a)]. The density values at both $\left(u_{1}, u_{2}\right)$ and $\left(-u_{1}, u_{2}+0.5\right)$ should be included in the kernel to construct the kernel starting from $(r, \phi)$. Thus the stochastic kernel expressed in polar coordinates is

$$
\begin{aligned}
& g_{A_{n}, I_{n}}(v, \psi ; r, \phi) \\
& \quad=\sum_{p=-\infty}^{p=+\infty}\left\{n\left[(\nu, \psi+p) ; \boldsymbol{U}_{I_{n}}^{(0)}(r, \phi), \epsilon^{2} \boldsymbol{\Sigma}_{I_{n}}\right]\right. \\
& \left.\quad+n\left[(-v, \psi+0.5+p) ; \boldsymbol{U}_{I_{n}}^{(0)}(r, \phi), \epsilon^{2} \boldsymbol{\Sigma}_{I_{n}}\right]\right\},
\end{aligned}
$$

where $v \in \boldsymbol{R}^{+}, \psi \in[0,1)$, and $\boldsymbol{U}_{I_{n}}^{(0)}(r, \phi)$ explicitly denote the initial values of $\boldsymbol{U}_{I_{n}}^{(0)}$ (it should be noted that $r$ and $\phi$ are
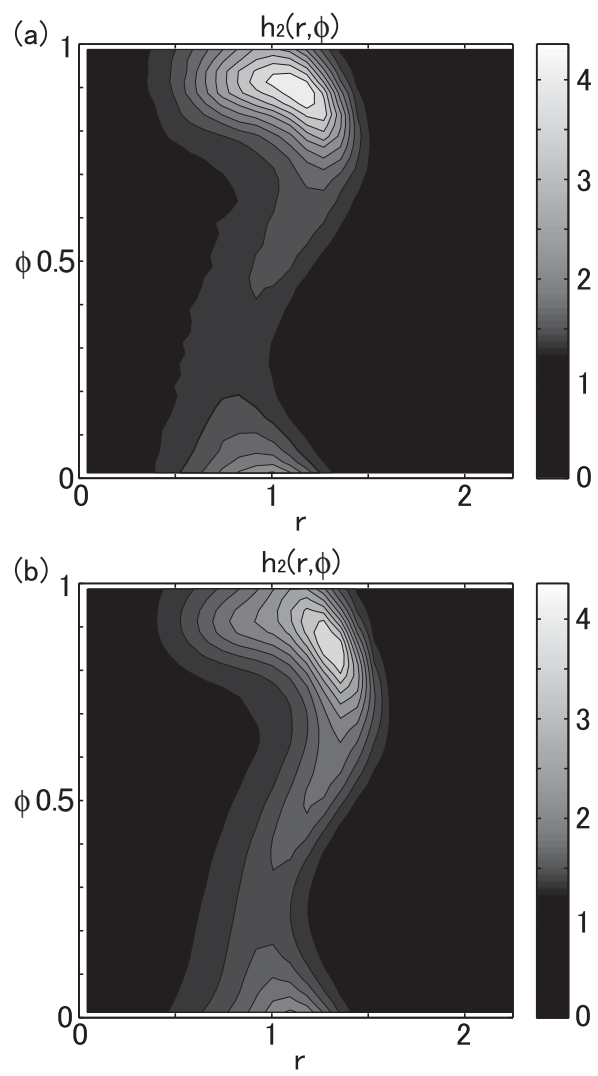

FIG. 4. (a) Density $h_{2}(r, \phi)$ obtained by the Euler method with a time step of 0.0001 and 500000 different sample paths in the $r-\phi$ plane. (b) Density obtained by the SPTO approximated by a $50 \times 50$ matrix in the $r-\phi$ plane. In both cases, the parameters used are $A=0.9, I_{1}=0.4, I_{2}=0.7$, and $k=1.0$.

included in the equations of $r^{\prime}$ and $\phi^{\prime}$, respectively). The summation in Eq. (8) takes into account the possibility of multiple rotations around the origin; the subscripts in $g_{A_{n}, I_{n}}$ represent the dependence of the kernel on the input parameters $A_{n}$ and $I_{n}$, respectively. Figure 2(b) shows an example of the kernel.

Finally, the Markov operator that expresses the relation between the density just before the $n$th impulse and that just before the $(n+1)$ th impulse is written as

$$
\begin{aligned}
h_{n+1}(v, \psi) & =\boldsymbol{P}_{A_{n}, I_{n}} h_{n}(\nu, \psi) \\
& =\int_{0}^{1} \int_{0}^{\infty} g_{A_{n}, I_{n}}(v, \psi ; r, \phi) h_{n}(r, \phi) d r d \phi,
\end{aligned}
$$

where $h_{n}$ is the density of the stochastic Poincare oscillator just before the $n$th impulse in polar coordinates. We call this operator a stochastic phase transition operator (SPTO), which is a generalization of the phase transition curve. It should be noted that the stochastic kernel of the SPTO is derived explicitly. This allows one to know the structure of the stochastic kernel, which cannot be known from the numerical methods used to calculate the density evolution. In the following section the properties of the SPTO are explained using the structure of Eq. (8). 
(a)
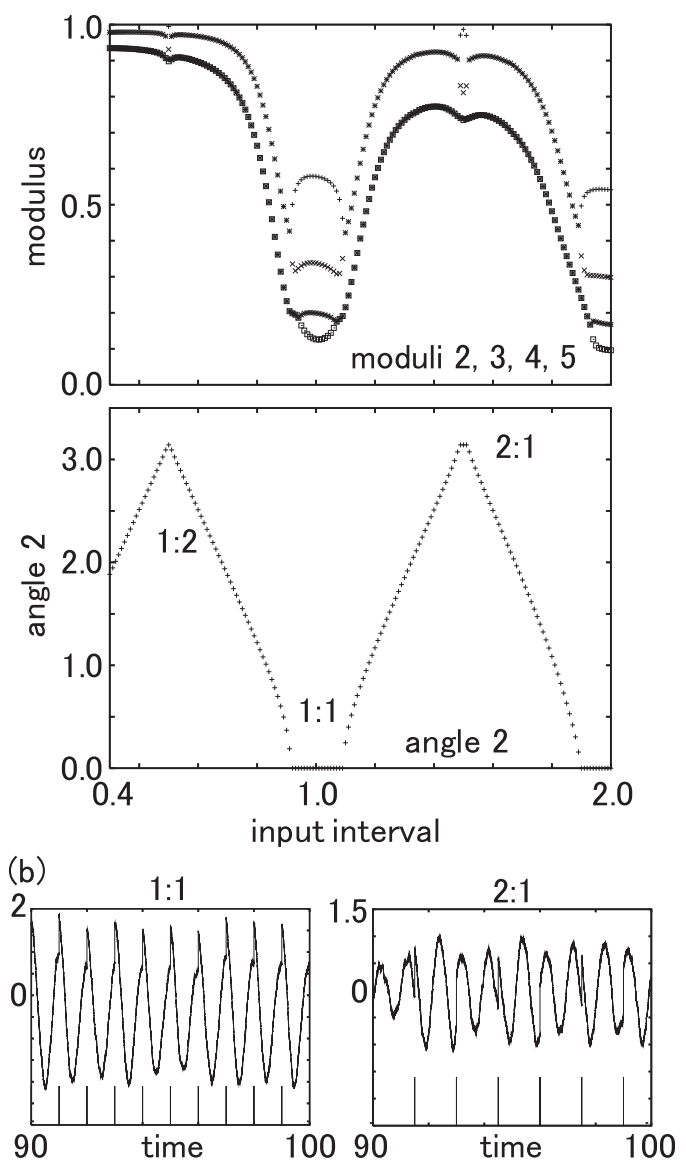

(c)
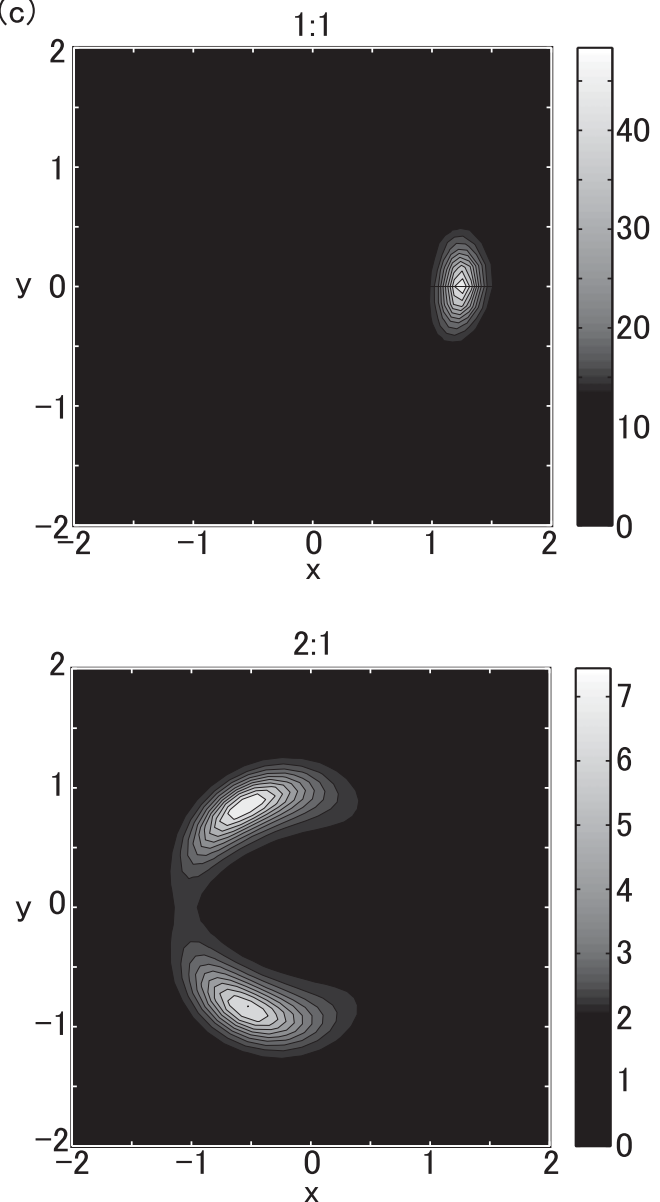

FIG. 5. (a) Moduli and angle of the eigenvalues as functions of the interimpulse interval. The abscissa plots the interimpulse interval in arbitrary units and the ordinate plots the angle in radians. The moduli of the second to fifth eigenvalues and the angle of the second eigenvalue are shown. The stochastic phase-locking regions are indicated. (b) Wave forms for 1:1 (left) and 2:1 (right) stochastic phase lockings. (c) Invariant densities for 1:1 (top) and 2:1 (bottom) stochastic phase lockings. The input parameters are $\epsilon=0.3, A=0.9, k=1.0$, and $I=0.5$ for $1: 1$ stochastic phase locking and $I=1.0$ for $2: 1$ stochastic phase locking.

\section{PROPERTIES OF THE STOCHASTIC PHASE TRANSITION OPERATOR}

The properties of the SPTO are now described in its most basic form. To analyze the SPTO's properties, the operator $\boldsymbol{P}_{A_{n}, I_{n}}$ is discretized. The analyzed properties in response to periodic impulses having a constant amplitude $A$ and interimpulse interval $I=t_{n+1}-t_{n}(n=1,2, \ldots)$ are summarized. Since the SPTO is a linear operator, its properties are determined by its eigenvalues and eigenfunctions. Let $\left\{\alpha_{i}\right\}$ and $\left\{e_{i}\right\}(i=1,2, \ldots$,$) be the eigenvalues of \boldsymbol{P}_{A, I}$, sorted in descending order according to their moduli, and the corresponding eigenfunctions, respectively. Since the transition kernel satisfies $g_{A, I}>0, \alpha_{1}$ is 1 ; the multiplicity of $\alpha_{1}$ is one and its corresponding eigenfunction has a unique invariant density $h^{*}$ with positive coordinates. This means that the SPTO is ergodic [10] and $h^{*}$ has stationary dynamics. Furthermore, $\left|\alpha_{i}\right|<1$ for all eigenvalues other than 1 . Therefore, these eigenvalues and their corresponding eigenfunctions have information about the transient dynamics of the operator $\boldsymbol{P}_{A, I}$. Using these properties, one can decompose the SPTO into two parts:

$$
\boldsymbol{P}_{A, I} h(r, \phi)=\boldsymbol{V}_{A, I} h(r, \phi)+\boldsymbol{Q}_{A, I} h(r, \phi),
$$

where $\boldsymbol{V}_{A, I}$ represents stationary dynamics, i.e., $\boldsymbol{V}_{A, I} h(r, \phi)=$ $h^{*}(r, \phi)$, and $\boldsymbol{Q}_{A, I}$ corresponds to transient dynamics [10].

Generally, if the stochastic Poincaré oscillator receives $n$ time-varying impulses whose amplitude and interimpulse interval depend on time, the density just before the $(n+1)$ th impulse becomes

$$
\begin{aligned}
& h_{n+1}(r, \phi)=h_{A_{n}, I_{n}}^{*}(r, \phi)+\prod_{i=1}^{n} \boldsymbol{Q}_{A_{i}, I_{i}} h_{1}(r, \phi) \\
& +\left[\boldsymbol{P}_{A_{n}, I_{n}} h_{A_{n-1}, I_{n-1}}^{*}(r, \phi)-h_{A_{n}, I_{n}}^{*}(r, \phi)\right. \\
& \quad+\sum_{j=1}^{n-2} \prod_{l=1}^{j} \boldsymbol{Q}_{A_{n-l+1}, I_{n-l+1}}\left\{\boldsymbol{P}_{A_{n-j}, I_{n-j}} h_{A_{n-j-1}, I_{n-j-1}}^{*}(r, \phi)\right. \\
& \left.\left.-h_{A_{n-j}, I_{n-j}}^{*}(r, \phi)\right\}\right] .
\end{aligned}
$$


Equation (11) can be derived by using only those properties that are inherent to the SPTO. The first term on the right-hand side is the invariant density of the operator at the last impulse. The transient dynamics of the product $\boldsymbol{P}_{A_{n}, I_{n}} \boldsymbol{P}_{A_{n-1}, I_{n-1}} \cdots \boldsymbol{P}_{A_{1}, I_{1}}$ are determined by the second and third terms. The second term represents the dependence of the present density on the initial density. The third term originates from the difference between invariant densities of adjacent SPTOs in the product.

\section{NUMERICAL RESULTS}

Figure 3 shows a comparison between the invariant densities obtained by the SPTO and those obtained by a simulation using the Euler method in the $r-\phi$ plane. It is clear that the SPTO can reproduce the invariant density obtained by the simulation. The SPTO captures the dynamics away from the limit cycle. Furthermore, to check whether or not the product of the SPTOs can reproduce the response to the time-varying impulses, the response of the oscillator to two impulses with different interimpulse intervals $I_{1}$ and $I_{2}$ is examined. In this case, the corresponding SPTOs are $\boldsymbol{P}_{A, I_{1}}$ and $\boldsymbol{P}_{A, I_{2}}$, respectively. Figure 4 shows a comparison between the densities obtained from the simulation using the Euler method and those obtained using the product $\boldsymbol{P}_{A, I_{2}} \boldsymbol{P}_{A, I_{1}}$ in the $r-\phi$ plane. It can be seen that the product of the SPTOs can reproduce the response of the oscillator to these impulses.

To understand the structure of the SPTO, the eigenvalues are investigated as a function of the interimpulse interval [Fig. 5(a)]. Since the second eigenvalue of $\boldsymbol{P}_{A, I}$ corresponds to the eigenvalue with the largest modulus of $\boldsymbol{Q}_{A, I}$, we focus on this second eigenvalue. Figure 5(a) demonstrates that the second eigenvalue as a function of the interimpulse interval becomes real abruptly at some points, which are termed stochastic bifurcation points [6]. Several $p: q$ stochastic phase-locking regions, where $p$ times rotations occur roughly per $q$ impulses [Fig. 5(b)], are observed in Fig. 5(a); the period of the second eigenvalue is determined from its angle. These structures are the basis for analyzing the response of the stochastic Poincare oscillator to time-varying impulses.

Figure 6 shows the eigenvalues of the product of the SPTOs corresponding to impulses with a monotonically increasing or decreasing interimpulse interval. Observation of the numerically calculated eigenvalues reveals that they are distinct at each moment. In this case, the second eigenvalue describes the contribution of the second eigenfunction-the largest transient component of the product of the SPTOs- to the dynamics of a given impulse-driven stochastic nonlinear system. Thus the second eigenvalue of the product of the SPTOs reflects the contribution of the past activity to the present density as shown in Eq. (11). The modulus of the second eigenvalue shows an overall decreasing trend with increasing number of impulses and its angle alternates between 0 and $\pi$ in the 2:1 stochastic phase-locking region. This alternation originates from $\boldsymbol{P}_{A, I}$ with the parameter of the 2:1 stochastic phase-locking region. This response is an extension of stochastic phase locking to the time-varying parameter and we call it dynamic stochastic phase locking. Bifurcations in
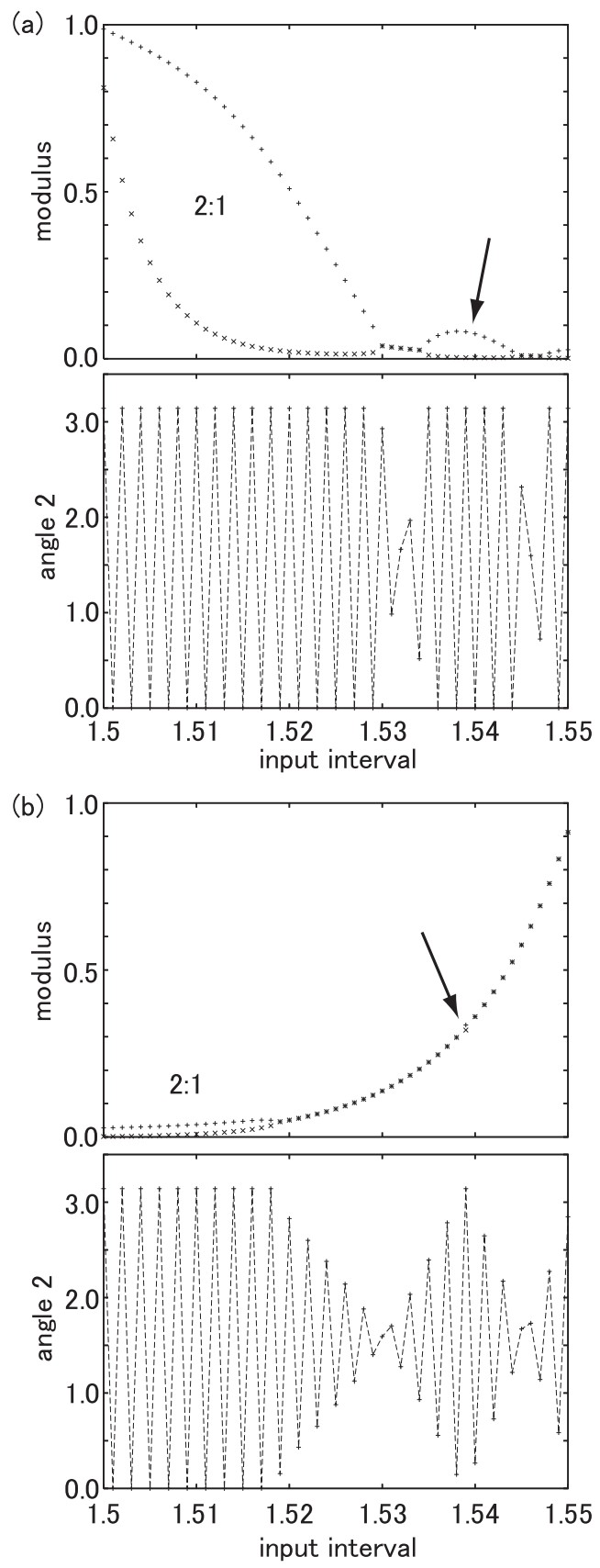

FIG. 6. Modulus and angle of the second eigenvalue of the product of SPTOs for (a) increasing and (b) decreasing inputs, where the interimpulse interval is given as $I_{n}=I_{1}+I_{\text {step }}(n-1)$. The abscissa plots the interimpulse interval in arbitrary units and the ordinate plots the angles in radians. The moduli of the second to fifth eigenvalues and the angle of the second eigenvalue are shown. The input parameters are (a) $\epsilon=0.3, A=0.9, k=1.0, I_{1}=1.5$, and $I_{\text {step }}=0.001$ and (b) $I_{1}=1.55$ and $I_{\text {step }}=-0.001$.

this region, which are an extension of the dynamic bifurcation [30], show hysteresis and delay, depending on the inputs. Furthermore, dynamic stochastic phase-locking regions that do not appear in the case of periodic impulses are also observed (indicated in Fig. 6 by arrows). These phenomena are possibly responsible for the hysteresis in the spike activity of pacemaker neurons [31]. 


\section{DISCUSSION}

History-dependent dynamics are observed at several levels of organization in nervous systems [32,33]. The second eigenvalue reflects the past activity of the system; in other words, it describes the degree to which the current state of the neuron model is affected by past activity and how the model conveys this historical information. This is particularly important since identification of the carrier of information implies determination of the range of past events that a spike can carry. Furthermore, this study presents an equation that clearly shows the relation between the neuron model's stochastic bifurcation structure and its response to arbitrary impulsive inputs.

In this study a SPTO for the stochastic Poincare oscillator was constructed. Since the small-disturbance asymptotic theory is not an ad hoc method, it is believed that the SPTO derived in this study can be generalized to other models such as the FitzHugh-Nagumo model. Further, the following can be achieved with the use of the present method, which is based on the small-disturbance asymptotic theory: (a) derivation of stochastic kernels systematically with the possibility of their improvement using higher-order expansion, and (b) definition of an appropriate product of the SPTOs for rapidly changing impulses. The generalization of the SPTO is beyond the scope of this paper.

\section{ACKNOWLEDGMENTS}

The author thanks Professor A. Takahashi for providing preprints. The author also thanks Professor K. Aihara for helpful comments. This work was supported by the Japan Science and Technology Agency, Precursory Research for Embryonic Science and Technology program.
[1] T. Yamanobe, K. Pakdaman, T. Nomura, and S. Sato, BioSystems 48, 287 (1998).

[2] M. Rabinovich, P. Varona, A. I. Selverston, and H. D. I. Abarbanel, Rev. Mod. Phys. 78, 1213 (2006).

[3] M. Rabinovich, R. Huerta, and G. Laurent, Science 321, 48 (2008).

[4] A. Hastings, Trends Ecol. Evol. 19, 39 (2004).

[5] T. Tateno, S. Doi, S. Sato, and L. M. Ricciardi, J. Stat. Phys. 78, 917 (1995).

[6] S. Doi, J. Inoue, and S. Kumagai, J. Stat. Phys. 90, 1107 (1998).

[7] F. Rieke, D. Warland, R. de Ruyter van Steveninck, and W. Bialek, Spikes, Exploring the Neural Code (MIT Press, Cambridge, MA, 1999).

[8] J. Segundo, M. Stiber, E. Altshuler, and J. Vibert, Neuroscience 62, 459 (1994).

[9] M. C. Mackey, Rev. Mod. Phys. 61, 981 (1989).

[10] A. Lasota and M. C. Mackey, Chaos, Fractals, and Noise: Stochastic Aspect of Dynamics, 2nd ed. (Springer-Verlag, Berlin, 1998).

[11] J. Losson and M. C. Mackey, Phys. Rev. E 52, 115 (1995).

[12] J. Losson and M. C. Mackey, Phys. Rev. E 52, 1403 (1995).

[13] T. Tateno, J. Stat. Phys. 92, 675 (1998).

[14] H. Nakao, K. Arai, K. Nagai, Y. Tsubo, and Y. Kuramoto, Phys. Rev. E 72, 026220 (2005).

[15] T. Shimokawa, K. Pakdaman, and S. Sato, Phys. Rev. E 59, 3427 (1999).

[16] T. Shimokawa, A. Rogel, K. Pakdaman, and S. Sato, Phys. Rev. E 59, 3461 (1999).
[17] T. Tateno and Y. Jimbo, Phys. Lett. A 271, 227 (2000).

[18] K. Pakdaman and D. Mestivier, Phys. Rev. E 64, 030901 (2001).

[19] T. Yamanobe and K. Pakdaman, Biol. Cybern. 86, 155 (2002).

[20] T. Tateno, Phys. Rev. E 65, 021901 (2002).

[21] W. H. Nesse, G. A. Clark, and P. C. Bressloff, Phys. Rev. E 75, 031912 (2007).

[22] P. C. Bressloff, Phys. Rev. A 44, 4005 (1991).

[23] H. Risken, The Fokker-Planck Equation, 2nd ed. (SpringerVerlag, Berlin, 1989).

[24] L. Glass and J. Sun, Phys. Rev. E 50, 5077 (1994).

[25] M. Stiber, K. Pakdaman, J. F. Vibert, E. Boussard, J. Segundo, T. Nomura, S. Sato, and S. Doi, BioSystems 40, 117 (1997).

[26] T. Nomura, S. Sato, S. Doi, J. Segundo, and M. D. Stiber, Biol. Cybern. 72, 93 (1994).

[27] N. Kunitomo and A. Takahashi, Ann. Appl. Probab. 13, 914 (2003).

[28] C. Gardiner, Stochastic Methods, 4th ed. (Springer-Verlag, 2009).

[29] A. Takahashi, K. Takehara, and M. Toda, CARF Working Paper, CARF-F-242 (2011).

[30] E. Benoit, Dynamic Bifurcations (Springer-Verlag, Berlin, 1991).

[31] J. Segundo, M. Stiber, J. Vibert, and S. Hanneton, Neuroscience 68, 693 (1995).

[32] G. Gilboa, R. Chen, and N. Brenne, J. Neurosci. 25, 6479 (2005).

[33] M. Saleh, K. Takahashi, Y. Amit, and N. Hatsopoulos, J. Neurosci. 30, 17079 (2010). 